\title{
An ethnobotanical study in Pöhrenk village (Çiçekdağı-Kırșehir province / Turkey)
}

\author{
Berfin Çelik' (1), Yeter Yeșil' (1) \\ 'Istanbul University, Faculty of Pharmacy, Department of Pharmaceutical Botany, Istanbul,Turkey
}

ORCID IDs of the authors: B.C. 0000-0002-4926-2066; Y.Y. 0000-0002-4458-7881

Cite this article as: Celik, B., \& Yesil, Y. (2020). An ethnobotanical study in Pöhrenk village (Çiçekdağı-Kırșehir province / Turkey). istanbul Journal of Pharmacy, 50 (2), 131-141.

\begin{abstract}
Background and Aims: This article presents important ethnobotanical information obtained in Pöhrenk village (ÇiçekdağıKırșehir) which has the experience of severe migration. The aim of this study is to gather traditional ethnobotanical knowledge of wild plants used in this village which is located in the Central Anatolia Region of Turkey, and to identify the uses and local names of these wild plants.

Methods: The ethnobotanical study was carried out in Pöhrenk village between July 2018 and June 2019. The information, including the traditional uses of wild plants, was obtained from local people through face to face interviews, and during this study, 36 people ( 25 female and 11 male) were interviewed. During this period, demographic characteristics of participants, names of the local plants, their utilized parts and preparation methods were investigated and recorded.

Results: A total of 51 wild taxa belonging to 23 families were collected. According to the obtained data, the plants are mostly used as food (32 taxa), traditional folk medicine ( 9 taxa), making goods ( 6 taxa) and fodder ( 4 taxa). Also, the most represented families are Rosaceae (21.56\%), Asteraceae (15.68\%), Lamiaceae (5.88\%) and Fabaceae (5.88\%). Furthermore, the study was compared with three ethnobotanical studies conducted in nearby regions.

Conclusion: The data obtained in this study provided clues to ethnobotanists (or botanists), pharmacologists, and perhaps future local development projects.
\end{abstract}

Keywords: Çiçekdağı, Ethnobotany, Kırșehir, Pöhrenk, Traditional knowledge

\section{INTRODUCTION}

Since ancient times, the importance of plants in human life has been a known fact (Bulut, 2015). Traditional plant knowledge has always been verbally transmitted from generation to generation. This important information, compiled with ethnobotanical studies, is valuable for conservation, and the establishment of the local and indigenous plant usages has significant benefits (Sõukand \& Pieroni, 2016).

Detailed ethnobotanical studies in Turkey were started since the beginning of the $19^{\text {th }}$ Century (Ertuğ, 2014). Turkey, with the number of taxa of around 12000, has a rich flora, and about 3,800 of these taxa are endemic. In addition to this, many different cultures also live together in Turkey (Güner, Aslan, Ekim, Vural, \& Babaç, 2012). Therefore, it has a great wealth both in terms of traditional use of plants and local names of plants (Erik \& Tarikahya, 2004). However, the traditional use of plants has been adversely affected due to migration from rural to urban areas and factors such as people's orientation to synthetic drugs.

Ethnobotanical studies and studies on folk medicinal plants were carried out in Kırşehir province and nearby regions (Ayandın, 2010; Han \& Bulut, 2012; Şenkardeş, 2014; Vural, Karavelioğulları, \& Polat, 1997). In addition, a previous ethnobotanical study on 
Çiçekdağı and the surroundings of Kırşehir was published (Vural et al., 1997). Although Pöhrenk village, which is our area of study, is located within the borders of Çiçekdağı, it does not cover this study due to its distance to the district.

The aim of this study is to conduct a detailed ethnobotanical study in Pöhrenk Village (Çiçekdağı / Kırşehir) to avoid the disappearance of ethnobotanical knowledge, to relay this knowledge to new generations and to provide resources for future scientific studies.

\section{MATERIALS AND METHODS}

\section{Study area}

This study was conducted in Pöhrenk village, in the Çiçekdağı district (Kırşehir Province), which is located in the Central Anatolia region of Turkey. Pöhrenk village is one of 44 villages in the Çiçekdağı district (Figure 1). This region belongs to the IranoTuranian Plant Geography Region and falls within the B-5 grid square according to the Grid classification system, developed by Henderson (1961). The geographical location of the study area is $39^{\circ} 25^{\prime} 56.8^{\prime \prime}$ North and $34^{\circ} 27^{\prime} 14.18^{\prime \prime}$ East. Its altitude is approximately 1150 meters. The average annual temperature in the province is $10.2^{\circ} \mathrm{C}$, and the annual rainfall is $420 \mathrm{~mm}$ (Climate Data, 2019).

Pöhrenk village is 60 kilometers away from the center of Kırşehir, and 22 kilometers from the center of Çiçekdağı (Çiçekdağı Governor, 2019). The village residents immigrated from Adıyaman (East of Turkey), by the Ottoman Empire settlement laws in 1865 (Yıldırım, Ceyhan Suvari, Işoğlu, \& Bozkurt, 2006).

\section{Socio-economic structures}

The economy of the region is based on agriculture and animal husbandry. Wheat, barley and sunflower are the most usual cultivated crops in the region (Kırşehir Governor, 2019). Additionally, sheep breeding is common due to the fact that the study area is a natural vegetation steppe (Çiçekdağı Governor, 2019). However, the unemployment rate has increased due to the decline in agriculture in recent years, and there has been a high volume of migration from the village to big cities in Turkey, such as Istanbul and Ankara, and to European countries, such as Germany and Austria.

\section{Interviews with native people}

A total of $69.44 \%$ female and $30.56 \%$ male informants were interviewed. The informants had varying levels of education, with $29.87 \%$ having no education, $46.77 \%$ having a primary level, $15.53 \%$ having a secondary level and only $7.83 \%$ having a tertiary level of education.

The interviews were conducted with local people without much difficulty because one author (B.Ç.) is local to the area and has relations there. A questionnaire was administered to the local people through face-to-face interviews. Interviews were conducted in the fields and houses. We visited the fields during all seasons.

The International Society of Ethnobiology Code of Ethics was taken into account in the interviews (ISE, 2006).

\section{Plant materials}

The field studies were carried out between May 2018 and August 2019. During this period, the collected plants were pressed

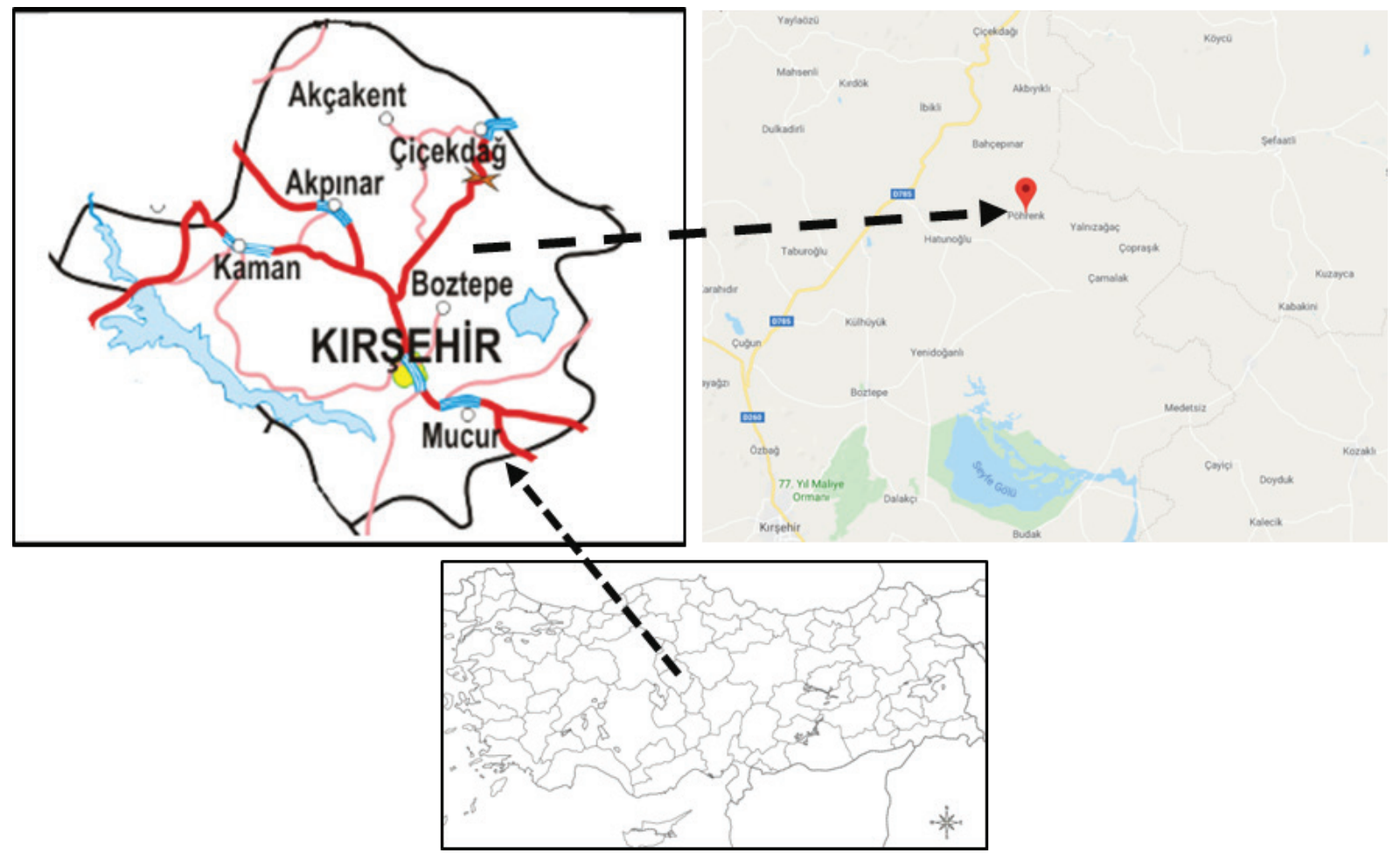

Figure 1. The location of Pöhrenk village in Turkey and Kırşehir. 
in the field and prepared for identification. These specimens were initially identified with the help of the Flora of Turkey (Davis, 1965-1985; 1988; Güner, Özhatay, Ekim, \& Başer, 2000), "A Checklist of the Flora of Turkey (Vascular Plants)" (Güner et al., 2012), "Illustrated Flora of Turkey Vol 1" (Güner et al., 2014) and "Illustrated Flora of Turkey Vol 2" (Güner et al., 2018) and "Türkiye'nin Doğal-Egzotik Ağaçları ve Çalıları" (Akkemik, 2018), and then they were compared with specimens in the Herbarium of the Faculty of Pharmacy of Istanbul University (ISTE). The scientific names of the plant taxa were identified according to "A Checklist of the Flora of Turkey (Vascular Plants)" (Güner et al., 2012). The plants were kept in ISTE.

\section{RESULTS}

The ethnobotanical knowledge about 51 taxa belonging 23 families was recorded. The local names of three taxa (Verbascum cheiranthifolium var. cheiranthifolium, Salvia dichroantha, Lotus corniculatus var. corniculatus) are unknown. The detailed knowledge including scientific name, voucher number, family name, life form, local name, used part(s), use, utilization method and preparation are summarized in Table 1. The most common families are Asteraceae (15.68\%), Rosaceae (21.56\%), Lamiaceae (5.88\%) and Fabaceae (5.88\%). The plants are used for food (32 taxa), traditional folk-medicine (9 taxa), making goods (6 taxa), fodder (4 taxa), firewood (4 taxa), ornament (2 taxa), cosmetic (1 taxon), fragrance (1 taxon) and evil eye (1 taxon). The percentages of plants' use are shown in Figure 2.

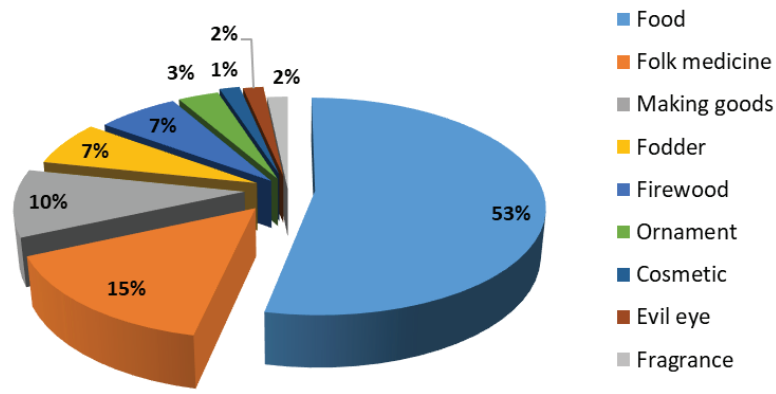

Figure 2. The percentages of plants' use in Pöhrenk village.
The edible plants are consumed eaten raw, prepared spice, soap, 'pilav' with bulgur, 'sarma', compote, marmalade, jam, 'şerbet' and tea, fried with onion or prepared as pancakes. Sarma is a cooked leaf rolled around a filling made from rice and/ or minced meat (Doğan, Nedelcheva, \& Pieroni, 2017). The use of the spice prepared with the leaves of Mentha longifolia is very common (Figure 3).

The most commonly used parts of plants are the aerial parts (19 taxa), fruits (11 taxa), leaves (8 taxa), flowers (4 taxa) and capitulums (2 taxa) (Figure 4). The aerial parts and leaves of raw consumed plants as food are usually collected in early April. Most of the plants whose fruits are consumed are in the Rosaceae family, and they are usually consumed raw or consumed as compote (Figure 5 and 6). The parts of all plants used as fodder are the aerial parts.

Additionally, the life forms of the used plants are herbs (68.62\%), trees (19.60\%) and shrubs (11.76\%), in descending order. It was reported that the most important plants were Polygonum cognatum, Teucrium polium, Malva neglecta, Mentha longifolia, Prunus cocomilia, P. divaricata and P. spinosa.

Three of the collected taxa are endemic. These taxa are Salvia dichroantha, Anchusa leptophylla subsp. incana and Crocus ancyrensis (Figure 7).

\section{DISCUSSION}

Ethnobotanical studies became widespread in Turkey at the beginning of the 90s, and more folk-medicinal uses were recorded in the studies conducted at that time. It is possible to observe the same feature in a previous study which was conducted in Çiçekdağı's center and its surroundings (Vural et al., 1997). When we compare our data with this study, 10 taxa (Chenopodium album, Crataegus orientalis, Gundelia tournefortii, Peganum harmala, Polygonaum cognatum, Potentilla reptans, Pyrus elaeagnifolia, Rosa canina, R. hemisphaerica, Teucrium polium) are common, and six of them (Polygonaum cognatum, Chenopodium album, Peganum harmala, Rosa canina, R. hemisphaerica, Teucrium polium) have the same use. Also, Gundelia tournefortii, Peganum harmala, Polygonaum cognatum and Rosa canina have the same local name. However, Rosa canina
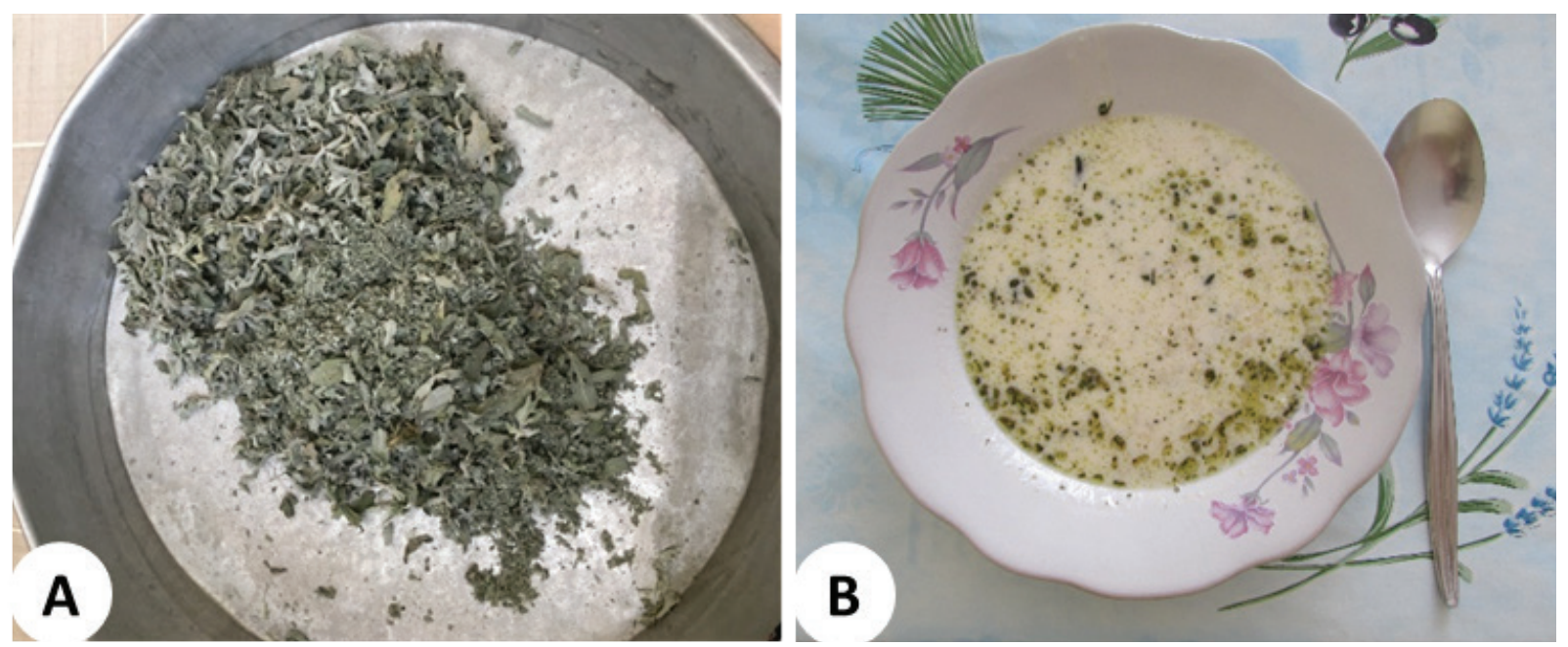

Figure 3. The spice of Mentha longifolia, A) dried leaves of M. longifolia, B) Yoghurt soup with its spice. 


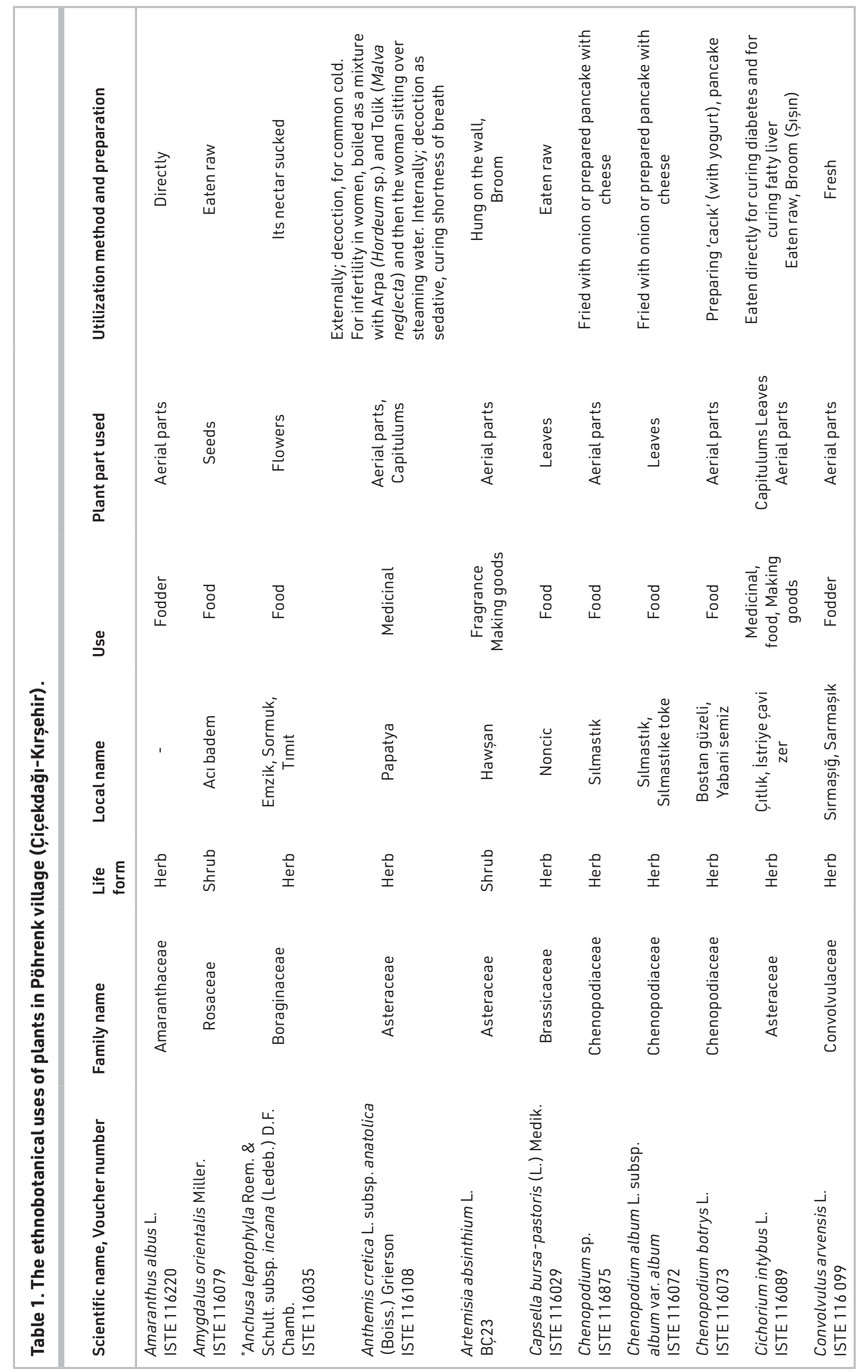




\begin{tabular}{|c|c|c|c|c|c|c|c|c|c|c|c|c|c|}
\hline 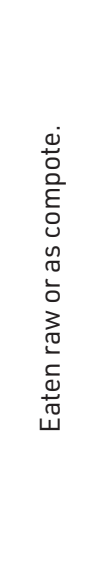 & 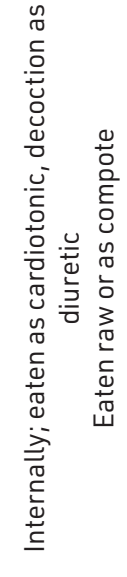 & 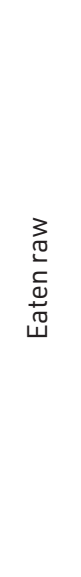 & $\begin{array}{l}\frac{c}{\hat{N}} \\
\text { L્L }\end{array}$ & 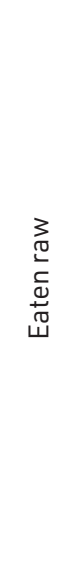 & 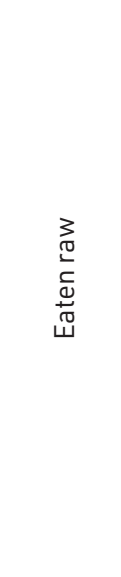 & 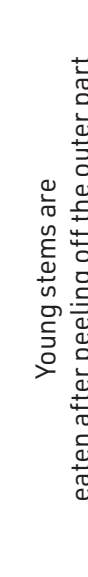 & & 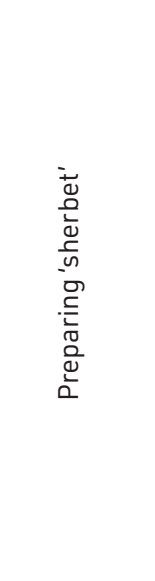 & 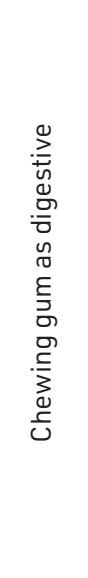 & 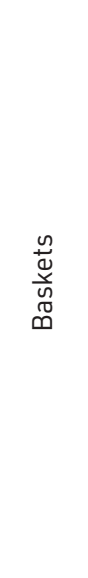 & 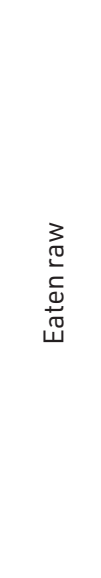 & 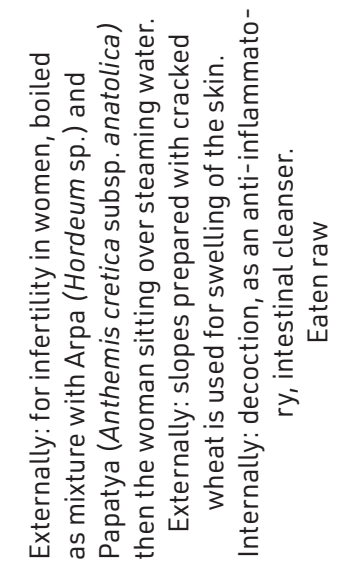 & 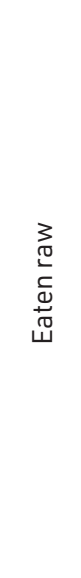 \\
\hline 点 & 芳 & 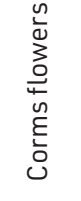 & 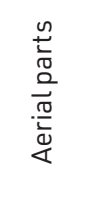 & $\begin{array}{l}\text { N } \\
\stackrel{0}{0} \\
\therefore\end{array}$ & 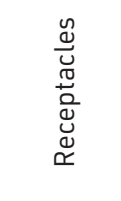 & $\begin{array}{l}E \\
\stackrel{\Xi}{\varpi} \\
\dot{\omega}\end{array}$ & & $\begin{array}{l}\text { 崩 } \\
3_{0}^{3} \\
\frac{0}{4}\end{array}$ & 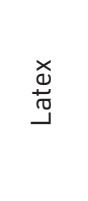 & $\begin{array}{l}\frac{n}{0} \\
\frac{0}{0} \\
\frac{0}{00} \\
\frac{0}{2} \\
\frac{0}{4}\end{array}$ & $\begin{array}{l}\stackrel{n}{0} \\
\mathbb{\Xi} \\
\sim\end{array}$ & $\begin{array}{l}\frac{n}{0} \\
\frac{0}{0} \\
\frac{0}{00} \\
\frac{0}{4} \\
\frac{\alpha}{\alpha}\end{array}$ & 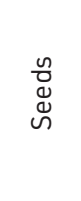 \\
\hline $\begin{array}{l}\text { ㅁ } \\
\text { 운 }\end{array}$ & 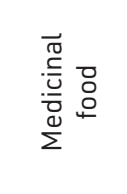 & $\begin{array}{l}\text { 뭉 } \\
\text { ○े }\end{array}$ & 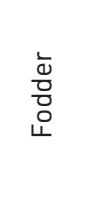 & 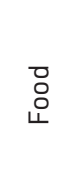 & $\begin{array}{l}\text { 응 } \\
\text { ○ }\end{array}$ & 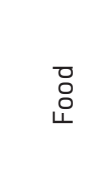 & & 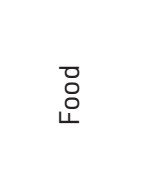 & 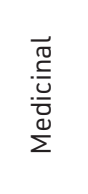 & 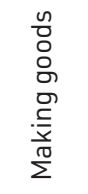 & 은 & 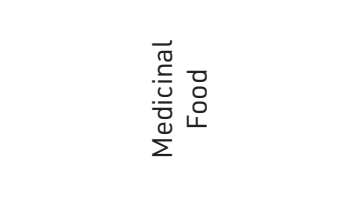 & $\begin{array}{l}\text { 뭉 } \\
\text { ○ }\end{array}$ \\
\hline 站 & 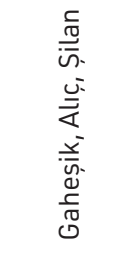 & $\begin{array}{l}\stackrel{x}{c} \\
\stackrel{D}{a} \\
\text { an }\end{array}$ & 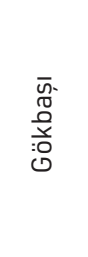 & 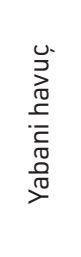 & 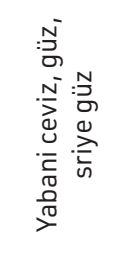 & 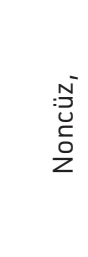 & & 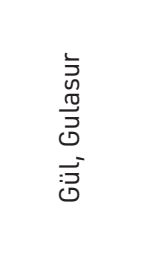 & $\begin{array}{l}\grave{\Phi} \\
\stackrel{\Xi}{\Phi} \\
\stackrel{\Phi}{\Phi}\end{array}$ & $\begin{array}{l}\text { Dू } \\
\text { 咅 } \\
\underline{\underline{\Sigma}}\end{array}$ & ' & 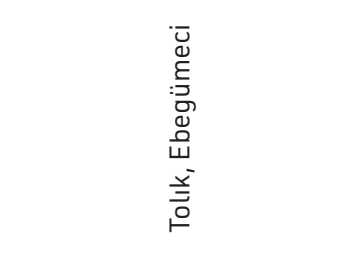 & 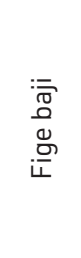 \\
\hline$\stackrel{\Xi}{\stackrel{\Xi}{ٌ}}$ & 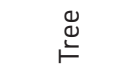 & $\begin{array}{l}\frac{0}{0} \\
\text { ㅁ }\end{array}$ & $\begin{array}{l}\frac{0}{\omega} \\
\frac{1}{I}\end{array}$ & 을 & 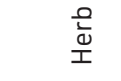 & 题 & & $\begin{array}{l}\stackrel{0}{0} \\
\stackrel{1}{1}\end{array}$ & $\begin{array}{l}\stackrel{0}{0} \\
\frac{0}{1}\end{array}$ & $\begin{array}{l}\stackrel{0}{0} \\
\frac{0}{1}\end{array}$ & $\begin{array}{l}\stackrel{0}{0} \\
\stackrel{0}{1}\end{array}$ & $\begin{array}{l}\frac{0}{\omega} \\
\text { I }\end{array}$ & 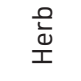 \\
\hline 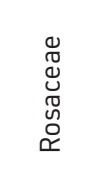 & 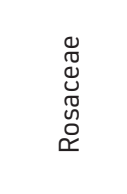 & $\begin{array}{l}0 \\
0 \\
0 \\
0 \\
0 \\
\frac{\pi}{0} \\
.0\end{array}$ & 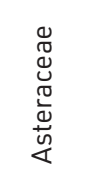 & 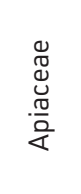 & 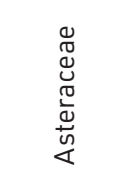 & $\begin{array}{l}0 \\
0 \\
0 \\
0 \\
.0 \\
\frac{\pi}{\alpha}\end{array}$ & & $\begin{array}{l}0 \\
\mathbb{0} \\
0 \\
0 \\
0 \\
0 \\
0 \\
0 \\
0 \\
0 \\
0\end{array}$ & 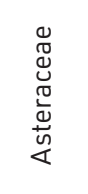 & 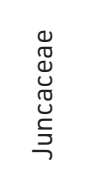 & 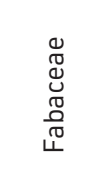 & 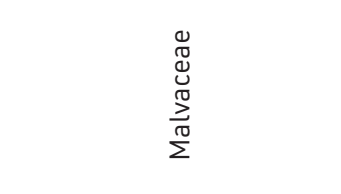 & 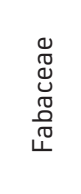 \\
\hline 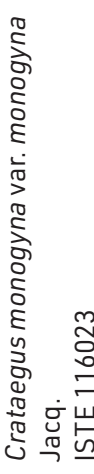 & 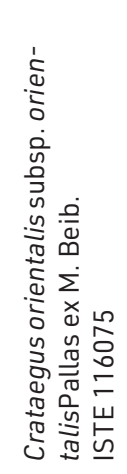 & 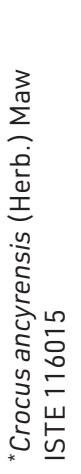 & 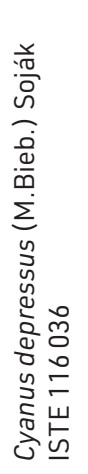 & 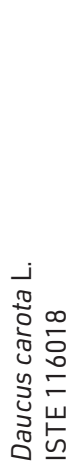 & 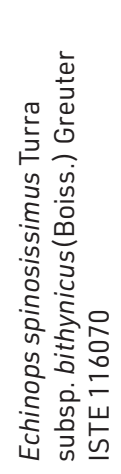 & 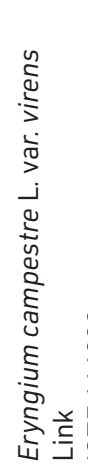 & 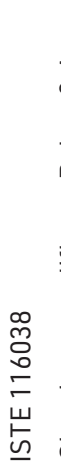 & 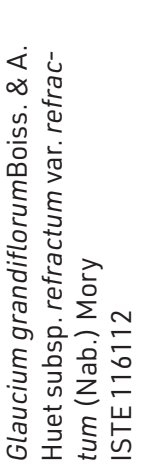 & 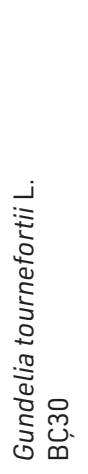 & 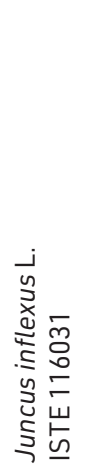 & 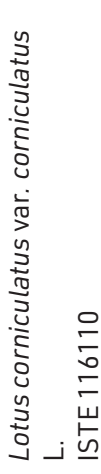 & 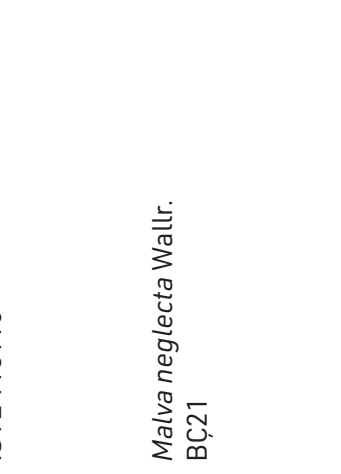 & 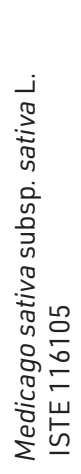 \\
\hline
\end{tabular}




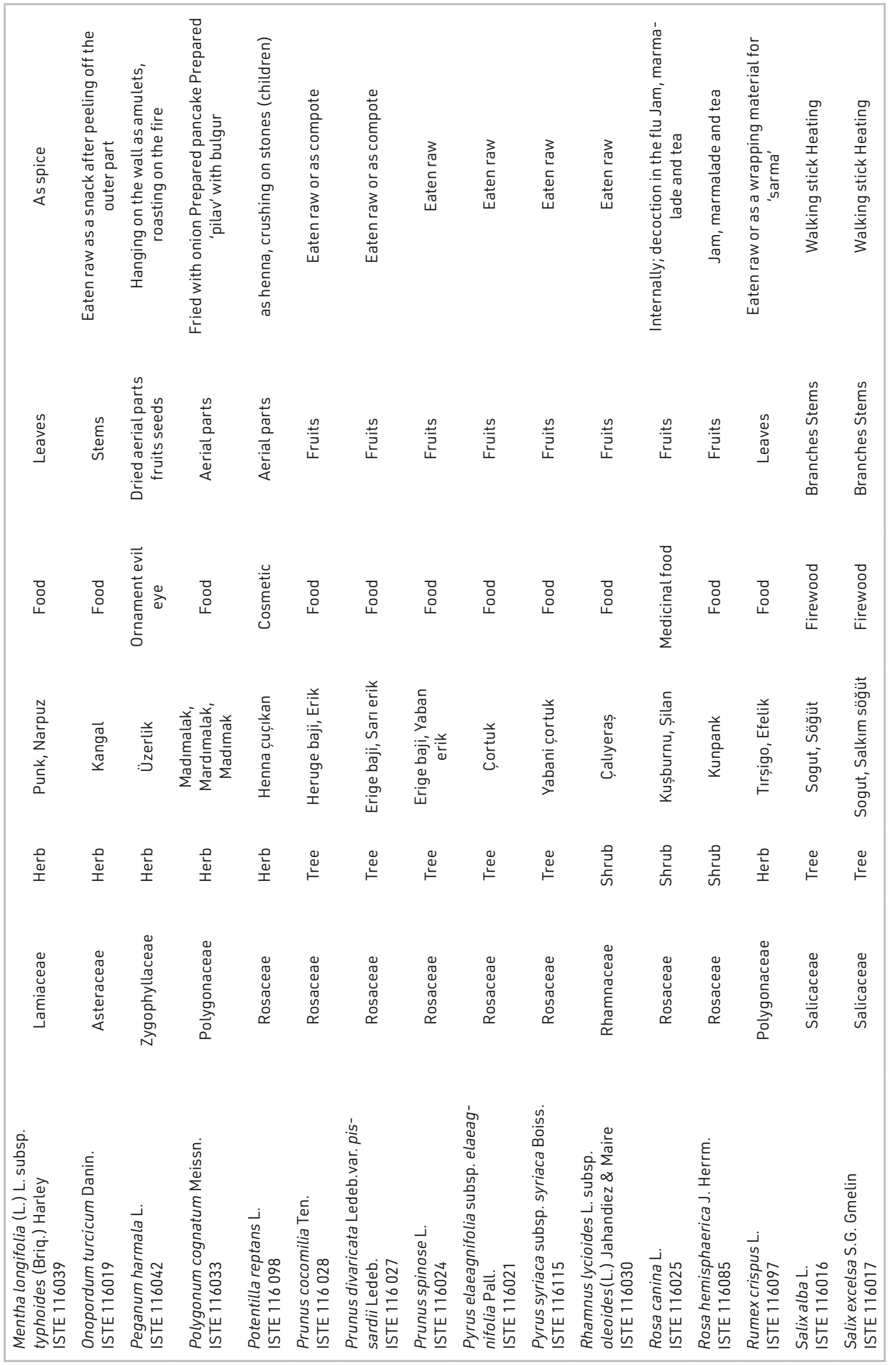




\begin{tabular}{|c|c|c|c|c|c|c|c|c|c|c|c|}
\hline 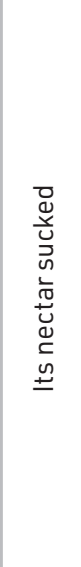 & $\begin{array}{l}\varepsilon \\
\text { ¿ } \\
\stackrel{0}{\infty}\end{array}$ & 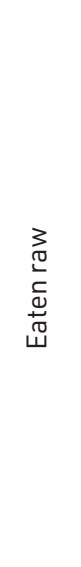 & $\begin{array}{l}\varepsilon \\
\stackrel{\Xi}{\circ} \\
\stackrel{0}{\infty}\end{array}$ & 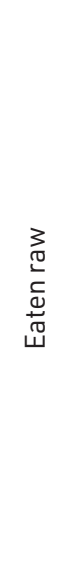 & 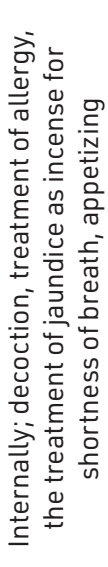 & 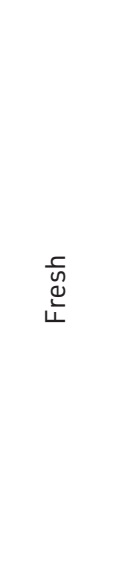 & 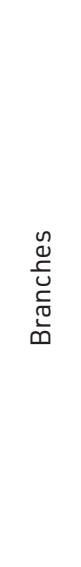 & 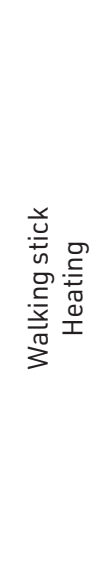 & 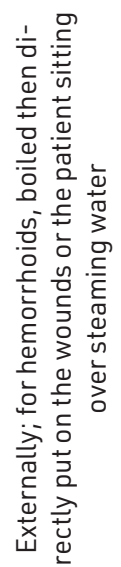 & 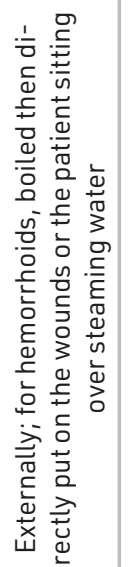 & \\
\hline $\begin{array}{l}\frac{n}{0} \\
\sum_{0}^{2} \\
\frac{0}{4}\end{array}$ & 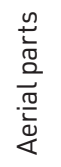 & 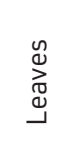 & 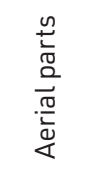 & $\begin{array}{l}\frac{0}{0} \\
\frac{0}{0} \\
\frac{0}{00} \\
\frac{\pi}{2} \\
\frac{0}{4}\end{array}$ & $\begin{array}{l}\frac{0}{0} \\
\frac{0}{0} \\
\frac{0}{0} \\
\frac{0}{0} \\
\frac{8}{4}\end{array}$ & 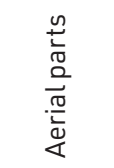 & $\begin{array}{l}\frac{n}{\sqrt[0]{0}} \\
\frac{0}{0} \\
\frac{\sigma 0}{2} \\
\frac{0}{4}\end{array}$ & 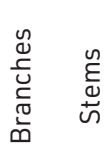 & 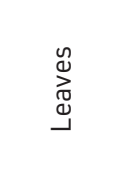 & 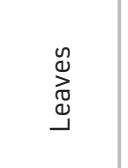 & \\
\hline $\begin{array}{l}\text { 무 } \\
\text { ○ }\end{array}$ & $\begin{array}{l}\frac{n}{0} \\
\dot{0} \\
0 \\
\frac{5}{5} \\
\frac{5}{\pi} \\
\frac{\pi}{2}\end{array}$ & 문 & 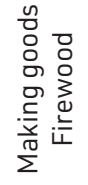 & $\begin{array}{l}\text { 무 } \\
\text { ㅇ }\end{array}$ & 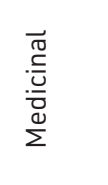 & $\begin{array}{l}\frac{\bar{d}}{2} \\
\overline{0} \\
\dot{0} \\
\dot{L}\end{array}$ & 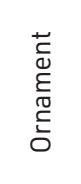 & 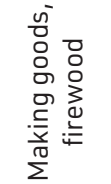 & 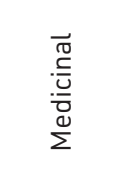 & 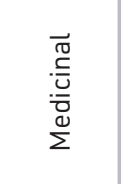 & \\
\hline & 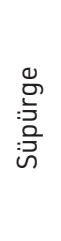 & 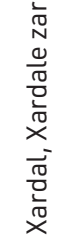 & 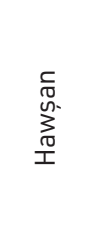 & 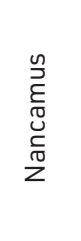 & $\begin{array}{l}\frac{\pi}{0} \\
\stackrel{0}{=} \\
=0\end{array}$ & $\begin{array}{l}\stackrel{0}{0} \\
\stackrel{0}{0}\end{array}$ & 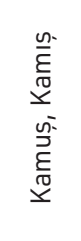 & 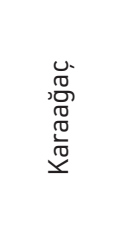 & ' & & \\
\hline $\begin{array}{l}\stackrel{0}{\frac{0}{ \pm}} \\
\text { I }\end{array}$ & $\begin{array}{l}\frac{0}{0} \\
\text { ㅁ }\end{array}$ & $\begin{array}{l}\frac{0}{\omega} \\
\frac{0}{I}\end{array}$ & $\frac{0}{\frac{2}{2}}$ & $\begin{array}{l}\frac{0}{0} \\
\text { ㅁ }\end{array}$ & $\begin{array}{l}\frac{0}{0} \\
\frac{0}{I}\end{array}$ & $\begin{array}{l}\text { 늠 } \\
\text { I }\end{array}$ & $\begin{array}{l}\frac{0}{0} \\
\frac{0}{I}\end{array}$ & 导 & $\begin{array}{l}\frac{0}{0} \\
\text { İ }\end{array}$ & 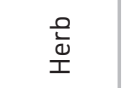 & \\
\hline 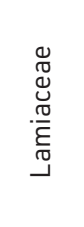 & 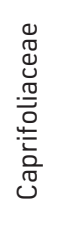 & 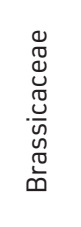 & 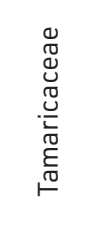 & 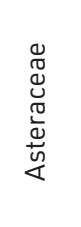 & 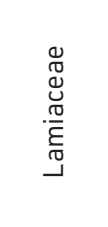 & 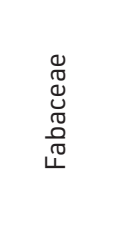 & 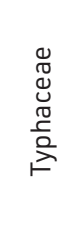 & $\begin{array}{l}0 \\
\widetilde{J} \\
\tilde{U} \\
\tilde{\sigma} \\
5 \\
5\end{array}$ & 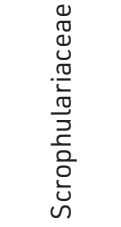 & 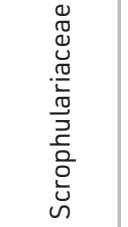 & \\
\hline 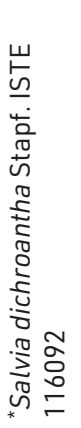 & 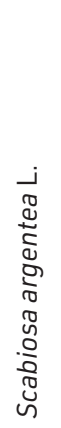 & 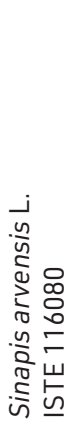 & 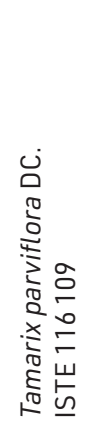 & 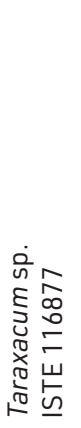 & 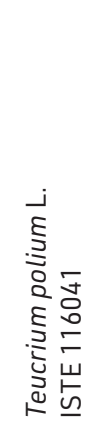 & 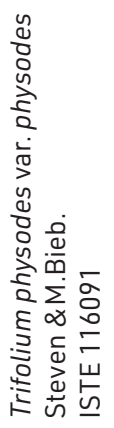 & 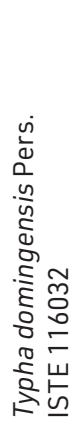 & 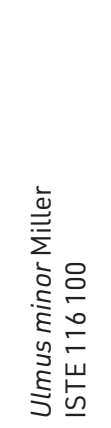 & 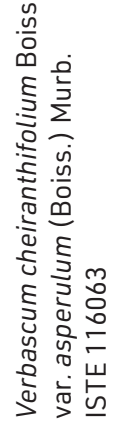 & 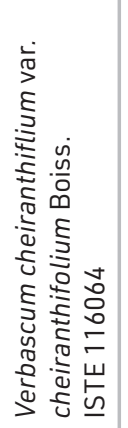 & 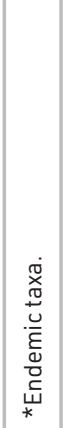 \\
\hline
\end{tabular}


is used for the treatment of flu in Pöhrenk, while it is used only as food and tea in the Çiçekdağı study. The use of Teucrium polium is, in general, the same in both regions, but differently, the plant is used for the treatment of jaundice in Pöhrenk. Peganum harmala, Potentilla reptans and Pyrus elaeagnifolia subsp. elaeagnifolia are used for medicinal purposes in Çiçekdağı, but these taxa are used for different purposes in Pöhrenk (Table 2).

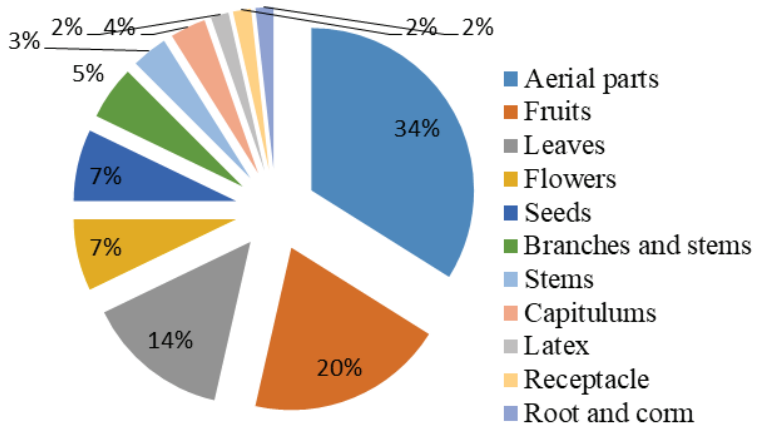

Figure 4. The percentages of plants used parts in Pöhrenk.

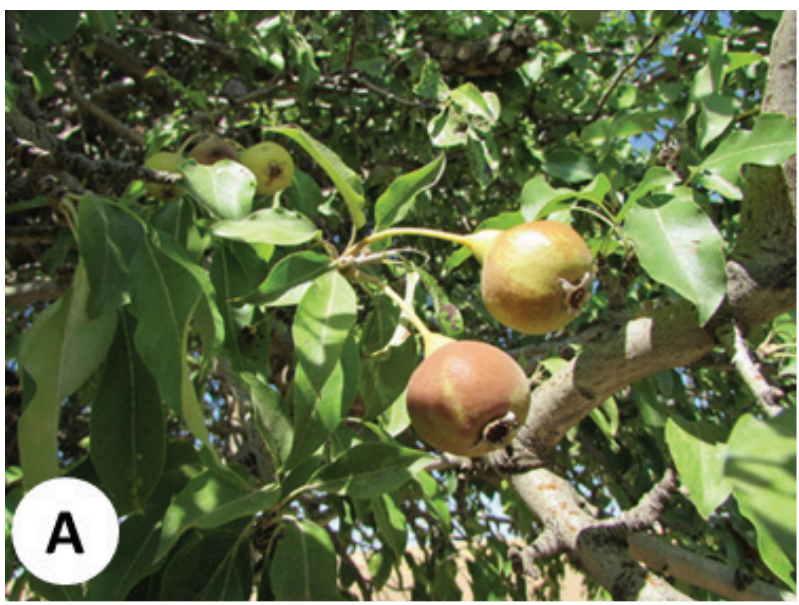

Figure 5. The fresh consumed fruits; A) Pyrus syriaca var. syriaca, B) Pyrus elaeagnifolia subsp. elaeagnifolia.

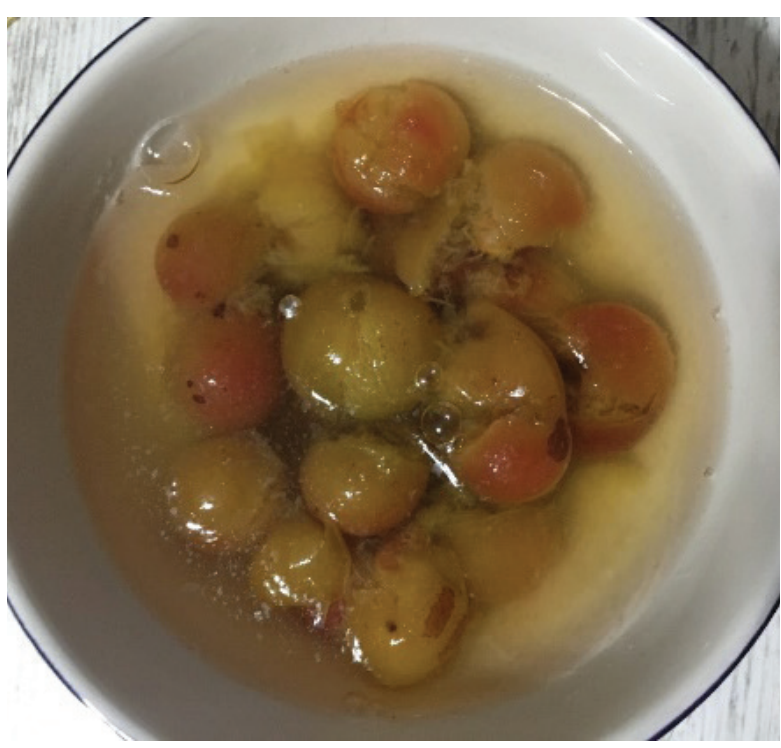

Figure 6. The compote of Prunus cocomilia and P. divaricata
The comparison of all the plants used in the present study with previous ethnobotanical studies (Ayandın, 2010; Han \& Bulut, 2012; Şenkardeş, 2014; Vural et al., 1997) in the nearby regions is given in Table 3 . Anthemis cretica subsp. anatolica, Chenopodium botrys and Verbascum cheiranthifolium are only used for medicinal purposes in Pöhrenk. Also, Chenopodium botrys, Crocus ancyrensis, Glaucium grandiflorum subsp. refractum var. refractum, Lotus corniculatus var. corniculatus, Mentha longifolia subsp. typhoides, Prunus cocomilia, Prunus spinosa, Pyrus syriaca, Rhamnus lycioides subsp. oleoides and Salvia dichroantha are only consumed as food in Pöhrenk. Additionally, the preparation of sherbet from Glaucium grandiflorum flowers is recorded only in Pöhrenk. However, Teucrium polium and Rosa canina are also used for medicinal purposes in nearby studies (Ayandın, 2010; Şenkardeş, 2014). Chenopodium album, Polygonum cognatum and Rosa canina are also consumed as food in tree nearby studies (Ayandın, 2010; Şenkardeş, 2014; Vural et al., 1997). Additionally, Peganum harmala is used for evil eye in both studies (Figure 8).
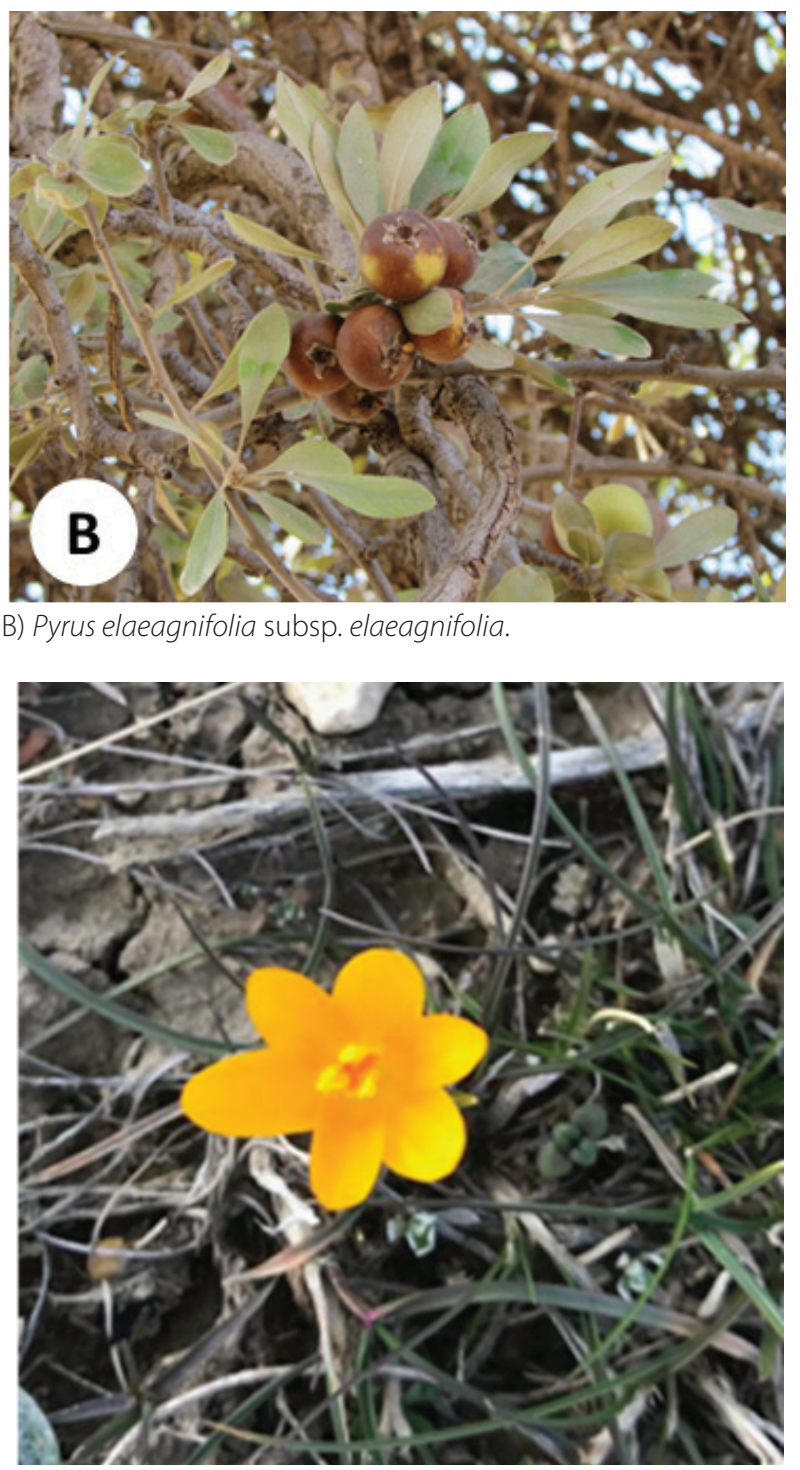

Figure 7. Crocus ancyrensis. 
Table 2. The comparison of the intended use of common plants in Pöhrenk Village and Çiçekdağı (Vural et al., 1997).

\begin{tabular}{|lll|}
\hline Scienific name & Intended use in Pöhrenk & Intended use in Çiçekdağı \\
\hline $\begin{array}{l}\text { Chenopodium album } \\
\begin{array}{l}\text { Crataegus orientalis subsp. } \\
\text { orientalis }\end{array}\end{array}$ & $\begin{array}{l}\text { Mood } \\
\text { diuretic) } \\
\text { Peganum harmala }\end{array}$ & Food \\
$\begin{array}{l}\text { Incense, evil eye, ornament } \\
\text { Potygonum cognatum }\end{array}$ & Food & Modicinal (skin diseases, hemorrhoids), evil eye \\
$\begin{array}{l}\text { Pyrus elaeagnifolia subsp. } \\
\text { elaeagnifolia }\end{array}$ & Cosmetic & Food \\
$\begin{array}{l}\text { Rosa canina } \\
\text { Rosa hemisphaerica }\end{array}$ & Food & Medicinal (antidiarrheal) \\
Teucrium polium & Food & Medicinal (blood purifier) \\
& Medicinal (allergy, appetizing, & Medicinal (appetizing, shortness of breath) \\
\hline
\end{tabular}

Table 3. The comparison of the intended use of common plants in Pöhrenk Village and in Çiçekdağı (Kırșehir) (Vural et al., 1997), Kadıșehri (Yozgat) (Han \& Bulut, 2012), Nevșehir (Acıgöl, Derinkuyú, Gülșehir, NevșehirMerkez, Ürgüp) (Șenkardeș, 2014) and Polatlı (Ankara) (Ayandın, 2010).

\begin{tabular}{|c|c|c|c|c|c|}
\hline Scientific name & $\begin{array}{l}\text { Pöhrenk } \\
\text { (Kırșehir) }\end{array}$ & $\begin{array}{l}\text { Çiçekdağı } \\
\text { (Kırșehir) }\end{array}$ & $\begin{array}{l}\text { Kadıșehri } \\
\text { (Yozgat) }\end{array}$ & Nevșehir & $\begin{array}{l}\text { Polatlı } \\
\text { (Ankara) }\end{array}$ \\
\hline Amaranthus albus & Fodder & - & - & - & - \\
\hline Amygdalus orientalis & Food & - & - & $\begin{array}{l}\text { Food, } \\
\text { medicinal }\end{array}$ & Food \\
\hline $\begin{array}{l}\text { *Anchusa leptophylla subsp. } \\
\text { incana }\end{array}$ & Food & - & - & Food & Food \\
\hline $\begin{array}{l}\text { Anthemis cretica subsp. } \\
\text { anatolica }\end{array}$ & Medicinal & - & - & - & - \\
\hline Artemisia absinthium & $\begin{array}{l}\text { Fragrance, } \\
\text { making good }\end{array}$ & - & - & - & - \\
\hline Capsella bursa-pastoris & Food & - & - & Food & - \\
\hline Chenopodium album & Food & Food & - & Food, medicinl & Food, fodder \\
\hline Chenopodium botrys & $\begin{array}{l}\text { Food, medicinal, } \\
\text { making good }\end{array}$ & - & - & - & - \\
\hline Cichorium intybus & Medicinal & - & - & $\begin{array}{l}\text { Food, } \\
\text { medicinal }\end{array}$ & Making good \\
\hline Convolvulus arvensis & Fodder & - & Medicinal & Fodder & Medicinal \\
\hline Crataegus monogyna & Food & $\begin{array}{l}\text { Food, } \\
\text { medicinal }\end{array}$ & - & $\begin{array}{c}\text { Food, } \\
\text { medicinal, } \\
\text { making good }\end{array}$ & - \\
\hline Crataegus orientalis & Food, Medicinal & - & - & $\begin{array}{c}\text { Food, } \\
\text { medicinal, } \\
\text { making good }\end{array}$ & $\begin{array}{l}\text { Food, making } \\
\text { good }\end{array}$ \\
\hline${ }^{*}$ Crocus ancyrensis & Food & - & - & - & - \\
\hline Cyanus depressus & Fodder & - & - & - & - \\
\hline Daucus carota & Food & - & - & - & Food, fodder \\
\hline $\begin{array}{l}\text { Echinops spinosissimus } \\
\text { subsp. bithynicus }\end{array}$ & Food & - & - & - & Fodder \\
\hline $\begin{array}{l}\text { Eryngium campestre var. } \\
\text { virens }\end{array}$ & Food & - & Medicinal & $\begin{array}{l}\text { Food, } \\
\text { medicinal }\end{array}$ & Making good \\
\hline
\end{tabular}




\begin{tabular}{|c|c|c|c|c|c|}
\hline $\begin{array}{l}\text { Glaucium grandiflorum subsp. } \\
\text { refractum }\end{array}$ & Food & - & - & - & - \\
\hline Gundelia tournefortii & Medicinal & - & - & $\begin{array}{l}\text { Food, medici- } \\
\text { nal }\end{array}$ & Food \\
\hline Juncus inflexus & Making good & - & - & - & - \\
\hline $\begin{array}{l}\text { Lotus corniculatus var. cor- } \\
\text { niculatus }\end{array}$ & Food & - & - & - & - \\
\hline Malva neglecta & Food, medicinal & - & Medicinal & $\begin{array}{c}\text { Food, me- } \\
\text { dicinal, making } \\
\text { good }\end{array}$ & Food \\
\hline Medicago sativa subsp. sativa & Food & - & - & - & Fodder \\
\hline $\begin{array}{l}\text { Mentha longifolia subsp. } \\
\text { typhoides }\end{array}$ & Food & - & - & $\begin{array}{l}\text { Food, medici- } \\
\text { nal }\end{array}$ & - \\
\hline Onopordum turcicum & Food & - & - & - & Food, fodder \\
\hline Peganum harmala & $\begin{array}{l}\text { Evil eye, orna- } \\
\text { ment }\end{array}$ & $\begin{array}{l}\text { Medicinal, evil } \\
\text { eye }\end{array}$ & Medicinal & $\begin{array}{l}\text { Medicinal, } \\
\text { making good, } \\
\text { evil eye }\end{array}$ & Evil eye \\
\hline Polygonum cognatum & Food & Food & Medicinal & Food & Food \\
\hline Potentilla reptans & Making good & Medicinal & - & - & - \\
\hline Prunus cocomilia & Food & - & - & - & - \\
\hline $\begin{array}{l}\text { Prunus divaricata subsp. } \\
\text { divaricata }\end{array}$ & Food & - & Medicinal & Food & - \\
\hline Prunus spinosa & Food & - & Medicinal & - & - \\
\hline Pyrus elaeagnifolia & Food & Medicinal & - & $\begin{array}{l}\text { Food, making } \\
\text { good }\end{array}$ & $\begin{array}{l}\text { Food, medici- } \\
\text { nal }\end{array}$ \\
\hline Pyrus syriaca & Food & - & - & - & - \\
\hline $\begin{array}{l}\text { Rhamnus lycioides subsp. } \\
\text { oleoides }\end{array}$ & Food & - & - & - & - \\
\hline Rosa canina & Food, medicinal & Food & Medicinal & $\begin{array}{l}\text { Food, medici- } \\
\text { nal }\end{array}$ & $\begin{array}{l}\text { Food, medici- } \\
\text { nal }\end{array}$ \\
\hline Rosa hemisphaerica & Food & Food & - & $\begin{array}{l}\text { Food, medici- } \\
\text { nal }\end{array}$ & Ornament \\
\hline Rumex crispus & Food & - & Medicinal & $\begin{array}{l}\text { Food, medici- } \\
\text { nal }\end{array}$ & - \\
\hline Salix alba & Firewood & - & Medicinal & $\begin{array}{l}\text { Medicinal, } \\
\text { making good, } \\
\text { fodder }\end{array}$ & Making good \\
\hline Salix excelsa & Firewood & - & - & - & - \\
\hline *Salvia dichroantha & Food & - & - & - & - \\
\hline Scabiosa argentea & Making good & - & - & - & - \\
\hline Sinapis arvensis & Food & - & - & $\begin{array}{l}\text { Food, medici- } \\
\text { nal }\end{array}$ & $\begin{array}{l}\text { Food, medici- } \\
\text { nal }\end{array}$ \\
\hline Tamarix parviflora & $\begin{array}{l}\text { Firewood, mak- } \\
\text { ing good }\end{array}$ & - & - & Firewood & - \\
\hline Teucrium polium & Medicinal & Medicinal & Medicinal & Medicinal & Making good \\
\hline $\begin{array}{l}\text { Trifolium physodes var. } \\
\text { physodes }\end{array}$ & Fodder & - & - & - & - \\
\hline Typha domingensis & Ornament & - & - & - & - \\
\hline Ulmus minor & Making good & - & - & Medicinal & $\begin{array}{l}\text { Evil eye, fire- } \\
\text { wood }\end{array}$ \\
\hline Verbascum cheiranthifolium & Medicinal & - & - & - & - \\
\hline
\end{tabular}



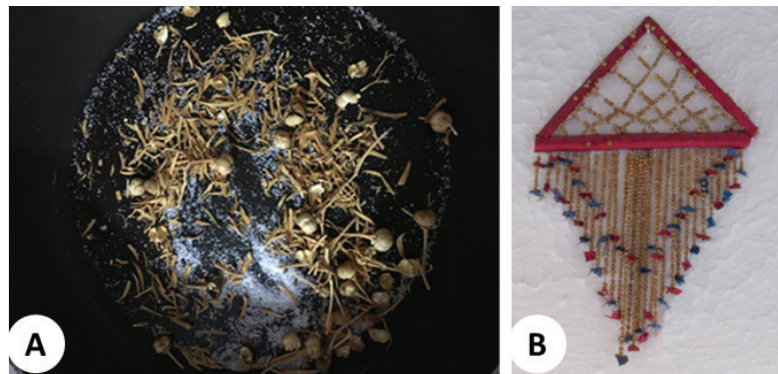

Figure 8. The use of Peganum harmala; A) Burning of $P$. harmala with salt to protect from evil eye, B) the ornament made of fruits of P. harmala.

Since the center of Çiçekdağı is close to the study area, it is expected that there will be more common plants compared to other regions, whereas fewer common plants are observed. The most common plants are observed in Nevşehir (24 taxa) and Polatlı (22 taxa).

\section{CONCLUSION}

The traditional knowledge is no longer being passed down from older to younger generations in Pöhrenk, because most of the residents (generally only the middle-aged and elderly) of the Pöhrenk village spend only the summer months in the village. The compiling of traditional ethnobotanical knowledge in this area is critical. This reveals the importance of this study, and this study will close the gap about traditional ethnobotanical knowledge.

Peer-review: Externally peer-reviewed.

Author Contributions: Conception/Design of Study- B.Ç., Y.Y.; Data Acquisition- B.Ç., Y.Y.; Data Analysis/Interpretation- B.Ç., Y.Y.; Drafting Manuscript-Y.Y.; Critical Revision of Manuscript- B.Ç., Y.Y.; Final Approval and Accountability- B.Ç., Y.Y.; Technical or Material Support- B.Ç., Y.Y.; Supervision- Y.Y.

Conflict of Interest: The authors have no conflict of interest to declare.

Financial Disclosure: Authors declared no financial support.

Acknowledgement: We thank to Hayrullah and Rukan Çelik for their help during the field trips. Also, we thank to the residents of Pöhrenk for their devoted helps.

\section{REFERENCES}

• Akkemik, Ü. (2018). Türkiyénin doğal-egzotik ağaçve çalıları (Gymnospermler-Angiospermler) [Turkey's natural-exotic trees and shrubs (Gymnospermae-Angiospermae)]. Ankara, Turkey: Orman Genel Müdürlüğü Yayınları.

- Ayandın, H. (2010). Ethnobotanical characteristics in the region between Avşar, Şabanözü and Çile Mount (Polatlı/Ankara) (Master of Science dissertation, Selçuk University, Institute of science, Konya). Retrieved from https://tez.yok.gov.tr/UlusalTezMerkezi/ tezSorguSonucYeni.jsp.

- Bulut, G., \& Tuzlacı, E. (2015). An ethnobotanical study of medicinal plants in Bayramiç (Çanakkale-Turkey). Marmara Pharmaceutical Journal, 19, 268-282.

- Merkel, A. (2019, May 15). Climate-data. Retrieved from https:// en.climate-data.org.
Davis, P. H. (Ed.) (1965-1985). Flora of Turkey and the East Aegean Islands (Vol. 1-9). Edinburgh: Edinburgh University Press.

Davis, P. H., Mill, R. R., \& Tan, K. (Eds.) (1988). Flora of Turkey and the East Aegean Islands (Vol. 10, Supplement I). Edinburgh: Edinburgh University Press.

- $\quad$ Doğan, Y., Nedelcheva, A., \& Pieroni, A. (2017). The diversity of plants used for the traditional dish sarma in Turkey: Nature, garden and traditional cuisine in the modern era. Emirates Journal of Food and Agriculture, 29, 429-440.

Erik, S., \& Tarıkahya, B. (2004). Türkiye florası üzerine [About flora of Turkey]. Kebikeç, 17, 139-163.

Ertuğ, F. (2014). Etnobotanik [Ethnobotany]. In A.Güner \& T. Ekim (Eds.), Resimli Türkiye florası [Illustrated Flora of Turkey] (Vol. 1, pp. 319-380). Istanbul, Turkey: Ali Nihat Gökyiğit Vakfı, Flora Araştırmaları Derneği ve Türkiye İ̧̧ Bankası Kültür Yayınları.

Given, D. R., \& Harris, W. (1994). Techniques and methods of ethnobotany. Lincoln: Commonwealth Secretariat.

- Güner, A., Özhatay, N., Ekim, T., \& Başer, K. H. C. (Eds.). (2000). Flora of Turkey and the East Aegean Islands (Vol. 11 Supplement II). Edinburgh: Edinburgh University Press.

Güner, A., Aslan, S., Ekim, T., Vural, M., \& Babaç, M. T. (Eds.). (2012). Türkiye bitkileri listesi (Damarlı bitkiler) [Turkey plant list (Vascular plants)]. Istanbul, Turkey: Flora Araştırmaları Derneği ve Nezahat Gökyiğit Botanik Bahçesi Yayınları.

Güner, A. (Ed.). (2014). Resimli Türkiye Florası cilt 1 [Illustrated Flora of Turkey vol. 1]. Istanbul: Nezahat Gökyiğit Botanik Bahçesi, Flora Araştırmaları Derneği ve Türkiye Iş̧ Bankası Kültür Yayınları.

- Güner, A., Kandemir, A., Menemen, Y., Yıldırım, H., Aslan, S., Ekşi, G., Güner, I. \& Çimen, A. Ö. (Eds.) (2018). Resimli Türkiye florası [lllustrated flora of Turkey] (Vol. 2). Istanbul, Turkey: Ali Nihat Gökyiğit Vakfı Nezahat Gökyiğit Botanik Bahçesi Yayınları.

- Han, M. I., \& Bulut, G. (2012). The flok-medicinal plants of Kadışehri (Yozgat). Acta Societatis Botanicorum Poloniae, 84(2), 237-248.

- Henderson, D. M. (1961). Contribution to the bryophyte flora of Turkey, IV. Notes from Royal Botanic Garden Edinburgh, 23, 263-278. International Society of Ethnobiology. (2019, Nov 18). The International society of ethnobiology code of ethics (with 2008 additions). Retrieved from http://www.ethnobiology.net/code-of-ethics/.

- Kendir, G., \& Güvenç, A. (2010). Ethnobotany and an overview on the ethnobotanical studies carried out in Turkey. Hacettepe Üniversitesi Eczacılık Fakültesi Dergisi, 30(1), 49-80.

- Kırşehir Governor. (2019, May 15). Retrieved from http://www. kirsehir.gov.tr.

- Çiçekdağı Governor. (2019, May 20). Retrieved from http://www. cicekdagi.gov.tr.

Sõukand, R., \& Pieroni, A. (2016). The importance of a border: Medical, veterinary, and wild food ethnobotany of the Hutsuls living on the Romanian and Ukrainian sides of Bukovina. Journal of Ethnopharmacology, 185, 17-40.

- $\quad$ Yıldırım, A., Ceyhan Suvari, Ç., İsoğlu, I.M., Bozkurt, T. (2006). Artakalanlar: Anadolu'dan etnik manzaralar, [Residuals: Ethnic landscapes from Anatolia]. Istanbul, Turkey: E yayınevi.

- S Şenkardeş, I. (2014). Ethnobotanical researches in southern districts of Nevşehir (Acıgöl, Derinkuyu, Gülşehir, Nevşehir-Merkez, Ürgüp) (Doctoral dissertation, Marmara University, Institute of Medical Sciences, Istanbul). Retrieved from https://tez.yok.gov.tr/UlusalTezMerkezi/tezSorguSonucYeni.jsp.

- Vural, M., Karavelioğulları, F. A., \& Polat, H. (1997). Ethnobotanical properties of Çiçekdağı (Kırşehir) and surroundings. Ot Sistematik Botanik Dergisi, 4(1), 117-124.

- $\quad$ Yıldırım, A., Ceyhan Suvari, Ç., İşoğlu, I.M., Bozkurt, T. (2006). Artakalanlar: Anadolu'dan etnik manzaralar, [Residuals: Ethnic landscapes from Anatolia]. Istanbul, Turkey: E yayınevi. 